\title{
Association between absolute lymphocyte count and overall mortality in patients with surgically resected gastric cancer
}

\author{
Se Jun Park ${ }^{1}$, Jinsoo Lee ${ }^{1}$, Hyunho Kim ${ }^{1}$, Kabsoo Shin ${ }^{1}$, MyungAh Lee ${ }^{1}$, Jae Myung Park², Myung-Gyu Choi ${ }^{2}$, \\ Cho Hyun Park ${ }^{3}$, Kyo Young Song ${ }^{3}$, Han Hong Lee ${ }^{3}$, and In-Ho Kim ${ }^{1}$
}

Divisions of ${ }^{1}$ Medical Oncology and ${ }^{2}$ Gastroenterology, Department of Internal Medicine, ${ }^{3}$ Department of Surgery, Seoul St. Mary's Hospital, College of Medicine, The Catholic University of Korea, Seoul, Korea

Received: October 28, 2019 Revised : February 20, 2020 Accepted: April 13, 2020

\section{Correspondence to} In-Ho Kim, M.D. Division of Medical Oncology, Department of Internal Medicine, Seoul St. Mary's Hospital, College of Medicine, The Catholic University of Korea, 222 Banpo-daero, Seocho-gu, Seoul 06591, Korea

Tel: +82-2-2258-6044

Fax: +82-2-599-3589

E-mail: ihkmd@catholic.ac.kr https://orcid.org/0000-0002-

0351-2074
Background/Aims: Lymphocytes are an important component of the cell-mediated immune system. As lymphopenia is reportedly associated with poor prognoses in patients with various cancers, we investigated this notion in patients who underwent curative gastrectomy.

Methods: We retrospectively analyzed the association between absolute lymphocyte count (ALC) and prognosis in patients with stage I-III gastric cancer who underwent curative surgical resection. Ever lymphopenic patients were defined as those with ALCs $<1,000 / \mu \mathrm{L}$ at any time post-diagnosis except within 30 days post-surgery. Adjusted multivariable regression models were used to evaluate the associations between lymphopenia and overall mortality, gastric cancer-specific mortality, and disease-free survival.

Results: We investigated 1,222 patients diagnosed between January 2011 and December 2015. Fifty-six patients (4.6\%) were lymphopenic at diagnosis and nearly one-quarter (24.8\%) were ever lymphopenic with a mean minimum ALC of 640/ $\mu \mathrm{L}$. Older age (odds ratio [OR], 1.02) and higher stage (stage III vs. I; OR, 3.01) were positively associated with ever lymphopenia. On multivariable analysis, ever lymphopenia predicted higher overall mortality (hazard ratio [HR], 1.83; $p=0.008$ ), higher gastric cancer-specific mortality (HR, 1.58; $p=0.048)$, and shorter disease-free survival (HR, 1.83; $p=0.006$ ). The 5 -year gastric cancer-specific mortality rates for ever- and never lymphopenic patients were 10.9\% and 3.7\%, respectively; their 5-year cumulative recurrence rates were $15.1 \%$ and $4.6 \%$, respectively.

Conclusions: This study demonstrate that ever lymphopenia is independent prognostic factor for overall mortality and recurrence in patients with potentially curable gastric cancer; hence, ALCs may be a biomarker for predicting the prognoses of patients with stage I-III gastric cancer who had curative gastrectomy.

Keywords: Gastric cancer; Peripheral lymphocyte count; Prognosis; Recurrence

\section{INTRODUCTION}

Gastric cancer is the fifth most frequently diagnosed cancer and the third leading cause of cancer-related death worldwide [1]. This disease is one of the most common cancers in East Asia, particularly in Korea and Japan. Surgery is the primary treatment option for patients with localized gastric cancer; however, the 5-year 
overall survival rate of patients with stage II-III surgically resected gastric cancer is less than 50\% [2].

Numerous factors, such as tumor stage, resection margin, lymph node metastasis, and tumor grade, influence the risks of recurrence and death in patients with gastric cancer [3]. Previous studies have shown that pretreatment- or treatment-related lymphopenia may be associated with poor survival outcomes in patients with various carcinomas, sarcomas, and lymphomas [4-8]. In addition to poor survival, pretreatment lymphopenia has been associated with poor response to chemotherapy in various metastatic solid tumors [9]. Bruckner et al. [10] reported that the pretreatment absolute neutrophil count (ANC), absolute lymphocyte count (ALC), and absolute monocyte count are prognostic indicators for patients with metastatic gastric cancer. Since then, additional studies have demonstrated that the neutrophil-to-lymphocyte ratio (NLR) or monocyte-to-lymphocyte ratio are associated with overall survival and can be used as prognostic predictors in patients with gastric cancer [11-13]. Moreover, an increased NLR during adjuvant chemotherapy may be used as a prognostic indicator in patients with stage II-III gastric cancer [14].

Although several studies have concluded that the NLR is a prognostic indicator, there is limited data available on whether the ALC has prognostic value in potentially curable gastric cancer. Recent studies have shown that ALC is significantly associated with tumor-infiltrating lymphocytes (TILs) and mortality [15-17]. In patients with stage I-III triple-negative breast cancer, higher post-diagnosis peripheral ALCs predicted prolonged survival [15]. Furthermore, there was a direct positive correlation between TILs and ALCs during neoadjuvant chemotherapy or chemoradiotherapy $[15,16]$. In patients with relatively early-stage gastric cancer after gastrectomy, preoperative peripheral ALCs predicted postoperative survival [18]. Based on these data, we postulated that the ALC might influence cancer outcomes through the host's immune response to cancer.

In this study, we investigated factors associated with lymphopenia and evaluated whether ALC was associated with recurrence and mortality in patients with gastric cancer who underwent potentially curative surgical resection.

\section{METHODS}

\section{Patients}

We reviewed the record of patients with histologically confirmed adenocarcinoma or poorly cohesive carcinoma of the stomach or gastroesophageal junction who underwent curative gastric resection at the Catholic University of Korea, Seoul St. Mary's Hospital between January 2011 and December 2015. All patients had undergone potentially curative resection with $\mathrm{D} 2$ lymph node dissection and had no macroscopic or microscopic evidence of remnant tumors. D2 lymph node dissection was defined according to the guidelines of the Japanese Research Society for the Study of Gastric Cancer [19]. Patients with available electronic medical records were eligible if they met the following criteria: (1) 19 years of age or older; (2) histologically confirmed gastric or gastroesophageal junction adenocarcinoma or poorly cohesive carcinoma; (3) pathological stage I-III according to the American Joint Committee of Cancer, 7 th edition [2]; (4) Eastern Cooperative Oncology Group (ECOG) performance status score o-2; (5) completely recovered from their operation without any complication by 30 days post-surgery; and (6) adequate bone marrow and organ function (hemoglobin $>9.0 \mathrm{~g} / \mathrm{dL}$; platelet count $>100,000 / \mathrm{mm}^{3}$; total bilirubin $<1.5$-fold higher than the upper normal limit; serum transaminase $<3$-fold higher than the upper normal limit; and creatinine $<1.5$-fold higher than the upper normal limit). Patients with the following conditions were excluded (1) received neoadjuvant chemotherapy; (2) history of another cancer within the past 5 years (except adequately treated in situ or non-melanoma skin cancers); (3) newly diagnosed with secondary malignancies after surgery for gastric cancer; and (4) history of human immunodeficiency virus infection, autoimmune disease that required treatment with immunosuppressive agents, and/or receipt of systemic corticosteroids 2 weeks prior to surgery.

\section{Surveillance}

For surveillance against tumor recurrence, all patients were evaluated every 3 months during the first year, every 6 months for the following 5 years, and annually thereafter. Imaging evaluations were performed using computed tomography and magnetic resonance imaging according to the aforementioned schedule and were 
analyzed by radiologists at our institution. Levels of carcinoembryonic antigen and carbohydrate antigen 19-9 were also checked at every visit. If evidence suggested the possibility of recurrence, additional imaging or biopsy studies were undertaken to investigate the presence of gastric cancer; any recurrent disease was confirmed by radiology and histology.

\section{Variable definitions}

Baseline lymphopenia was defined as having an ALC < $1,000 / \mu \mathrm{L}$ within 2 weeks before surgery. If several preoperative ALC values were obtained, the count on the date closest to the surgery was considered. Ever lymphopenic and ever neutropenic were defined as having an ALC < $1,000 / \mu \mathrm{L}$ and an $\mathrm{ANC}<1,000 / \mu \mathrm{L}$, respectively, any time after the gastric cancer diagnosis. However, the following readings were excluded (1) those taken between the day of surgery and 30 days thereafter; (2) those acquired after the confirmation of gastric cancer recurrence; (3) those taken from patients during their last hospitalization if their admittance and death were for reasons other than gastric cancer; and (4) those taken during hospitalization for major surgery for reasons other than gastric cancer.

Overall mortality (OM) was defined as the percentage of patients who died of any cause during the study period. Gastric cancer-specific mortality (GCM) was defined as the percentage of patients who died due to gastric cancer during the study period. Disease-free survival (DFS) was defined as the interval between curative surgery and recurrence, death, or the date of the last follow-up.

\section{Statistical analysis}

A logistic regression model was used to determine whether baseline lymphopenia or ever lymphopenia was associated with age at diagnosis, cancer stage, human epidermal growth factor receptor 2 overexpression or amplification status, adjuvant chemotherapy administration, and ever neutropenia. Odds ratios (ORs) and 95\% confidence intervals (CIs) were estimated. Variables with a $p<0.05$ on the univariate analysis were subjected to multivariate analysis.

OM, GCM, and DFS for ever lymphopenic and never lymphopenic patients were calculated using Kaplan-Meier curves; differences between survival curves were tested for statistical significance using a two-tailed log-rank test. A multivariate Cox proportional hazards regression model was used to identify the effects of ever lymphopenia on survival. Data from patients who were alive on the last follow-up date were censored. Hazard ratios (HRs) and 95\% CIs were calculated. All tests were two-sided and $p<0.05$ were considered statistically significant. Statistical analyses were performed using IBM version 24.o (IBM Co., Armonk, NY, USA) and GraphPad Prism version 8.o (GraphPad Software Inc., San Diego, CA, USA).

\section{Ethics}

This study was approved by the Institutional Review Board of the Catholic University of Korea, Seoul St. Mary's Hospital (IRB No: KC19RESIo292), and was performed in accordance with Korean regulations and the Declaration of Helsinki. The Institutional Review Board waived informed consent for this study due to the retrospective nature of the analysis.

\section{RESULTS}

\section{Patient characteristics}

In total, 1,222 patients with stage I-III gastric cancer were eligible for inclusion in this study. The baseline characteristics of these patients are shown in Table 1. The mean age at diagnosis was 59 years, and two-thirds of the patients (63.7\%) were male. Most patients (95.8\%) had good performance status (o-1), and 74.1\%, 13.8\%, and $12.1 \%$ of the patients had stage I, II, and III disease, respectively. Most patients had tubular adenocarcinoma $(82.6 \%)$, and the most common tumor location was the lower third of the stomach (62.8\%). One-quarter of the patients $(25.0 \%)$ received adjuvant chemotherapy; most (93.9\%) were never neutropenic, although nearly one-quarter (24.8\%) were ever lymphopenic, with a minimum ALC of $640 / \mu \mathrm{L}$ (Table 1). Over a median follow-up time of 4.9 years, 82 patients (6.7\%) died, 65 (5.3\%) of gastric cancer.

\section{Factors associated with lymphopenia}

ECOG performance status and cancer stage were associated with baseline lymphopenia after univariate analysis. After multivariate analysis, a higher cancer stage (stage III vs. I: OR, 3.81; 95\% CI, 2.05 to 7.09; $p<0.001$ ) 
Table 1. Patient demographics and reported ALCs at any time after gastric cancer diagnosis $(n=1,222)$

\begin{tabular}{|c|c|}
\hline Variable & Value \\
\hline Age in year & $59 \cdot 0 \pm 12$ \\
\hline \multicolumn{2}{|l|}{ Sex } \\
\hline Male & $778(63.7)$ \\
\hline Female & $444(36.3)$ \\
\hline \multicolumn{2}{|l|}{ ECOG performance status } \\
\hline $0-1$ & $1,171(95.8)$ \\
\hline 2 & $51(4.2)$ \\
\hline \multicolumn{2}{|c|}{ American Joint Committee on Cancer Stage ${ }^{a}$} \\
\hline I & $905(74.1)$ \\
\hline II & $169(13.8)$ \\
\hline III & $148(12.1)$ \\
\hline \multicolumn{2}{|l|}{ Pathological type } \\
\hline Tubular adenocarcinoma & $1,009(82.6)$ \\
\hline Poorly cohesive carcinoma & $213(17.4)$ \\
\hline \multicolumn{2}{|l|}{ Primary tumor location } \\
\hline EG junction & $20(1.6)$ \\
\hline Upper $1 / 3$ & $154(12.6)$ \\
\hline $\operatorname{Mid} 1 / 3$ & $269(22.0)$ \\
\hline Lower $1 / 3$ & $767(62.8)$ \\
\hline Diffuse & $12(0.9)$ \\
\hline \multicolumn{2}{|l|}{ HER2 amplification status } \\
\hline Amplified & $73(6.0)$ \\
\hline Negative & $919(75.2)$ \\
\hline Untested & $230(18.8)$ \\
\hline \multicolumn{2}{|l|}{ Received adjuvant chemotherapy } \\
\hline Yes & $305(25.0)$ \\
\hline No & $917(75.0)$ \\
\hline \multicolumn{2}{|l|}{ Baseline lymphopenia } \\
\hline Yes & $56(4.6)$ \\
\hline No & $1,166(95.4)$ \\
\hline \multicolumn{2}{|l|}{ Ever lymphopenia } \\
\hline Yes & $303(24.8)$ \\
\hline No & $919(75.2)$ \\
\hline Minimum ALC after diagnosis ${ }^{\mathrm{b}}, \mathrm{K} / \mu \mathrm{L}$ & $0.64 \pm 0.24$ \\
\hline \multicolumn{2}{|l|}{ Baseline neutropenia } \\
\hline Yes & $2(0.2)$ \\
\hline No & $1,220(99.8)$ \\
\hline \multicolumn{2}{|l|}{ Ever neutropenia } \\
\hline Yes & $74(6.1)$ \\
\hline No & $1,148(93.9)$ \\
\hline
\end{tabular}

Values are presented as mean \pm standard deviation or number (\%).

ALC, absolute lymphocyte count; ECOG, Eastern Cooperative Oncology Group; EG, esophagogastric; HER2, epidermal growth factor receptor 2 .

${ }^{\mathrm{a}}$ According to American Joint Committee on Cancer 7 th edition.

${ }^{\mathrm{b}}$ Definition of lymphopenia: ALC $<1,000 / \mu \mathrm{L}$. was found to be independently associated with baseline lymphopenia (Supplementary Table 1). Age at diagnosis, ECOG performance status, adjuvant chemotherapy administration, cancer stage, and ever neutropenia were associated with ever lymphopenia by univariate analysis; however, multivariate analysis revealed that only an older age at diagnosis (OR, 1.02; 95\% CI, 1.01 to 1.04; $p=$ 0.001), higher stage (stage III vs. I: OR, 3.01; 95\% CI, 1.87 to $4.84 ; p<0.001$ ), and ever neutropenia (OR, 3.51; $95 \%$ CI, 2.08 to $5.93 ; p<0.001$ ) were independently associated with ever lymphopenia (Table 2). The incidence of ever lymphopenia in stage I, stage II, and stage III patients with gastric cancer was $19.0 \%, 34.3 \%$, and $49.3 \%$, respectively (Supplementary Table 2). Only higher cancer stage was positively associated with both baseline and ever lymphopenia.

\section{Lymphopenia, OM, and GCM}

On multivariable analysis, patients with ever lymphopenia had significantly higher OM than that of never lymphopenic patients (HR, 1.83; 95\% CI, 1.17 to2.85; $p=$ 0.008 ) (Table 3 and Fig. 1A). Other predictors of increased OM were higher stage (stage III vs. I: HR, 33.8; 95\% CI, 17.1 to 66.7; $p<0.001$ ) and older age at diagnosis (HR, 1.05; 95\% CI, 1.02 to 1.07; $p<0.001$ ). Receiving adjuvant chemotherapy was associated with lower OM (HR, 0.50; 95\% CI, 0.29 to $0.88 ; p=0.017$ ). The 5 -year $\mathrm{OM}$ rate among ever lymphopenic patients was $13.4 \%$, whereas that among never lymphopenic patients was $4.4 \%$ (Fig. 1A).

Patients with ever lymphopenia also had significantly higher GCM(HR, 1.58; $95 \%$ CI, o.96 to 2.6o; $p=0.048$ ) (Table 3 and Fig. $1 \mathrm{~B}$ ) compared to that of never lymphopenic patients. Higher stage (stage III vs. I: HR, 158.7; 95\% CI, 55.5 to $471 ; p<0.001)$ and older age at diagnosis (HR, 1.03: $95 \%$ CI, 1.00 to $1.05 ; p=0.030$ ) were also associated with higher GCM, whereas receiving adjuvant chemotherapy was associated with lower GCM (HR, 0.37; 95\% CI, 0.20 to $0.66 ; p=0.001$ ). The 5-year GCM rate among ever lymphopenic patients was $10.9 \%$, whereas that among never lymphopenic patients was $3.7 \%$ (Fig. 1B).

\section{Lymphopenia and recurrence}

Univariate analysis showed that age at diagnosis, ECOG performance status, cancer stage, adjuvant chemotherapy administration, and ever lymphopenia were risk factors for gastric cancer recurrence. However, only high- 
Table 2. Univariate and multivariate analyses of predictors of ever lymphopenia $(\mathrm{ALC}<1,000 / \mu \mathrm{L} ; \mathrm{n}=1,222)$

\begin{tabular}{|c|c|c|c|c|}
\hline \multirow{3}{*}{ Variable } & \multicolumn{4}{|c|}{ Ever lymphopenia } \\
\hline & \multicolumn{2}{|c|}{ Univariate analysis } & \multicolumn{2}{|c|}{ Multivariate analysis } \\
\hline & OR $(95 \% \mathrm{CI})$ & $p$ value & OR $(95 \% \mathrm{CI})$ & $p$ value \\
\hline Age at diagnosis, /yr & $1.02(1.01-1.03)^{\mathrm{a}}$ & $<0.001^{\mathrm{a}}$ & $1.02(1.01-1.04)^{\mathrm{a}}$ & $0.001^{\mathrm{a}}$ \\
\hline ECOG 2 (vs. ECOG o-1) & $2.84(1.61-5.01)$ & $<0.001$ & $1.80(0.95-3.38)$ & 0.070 \\
\hline Received adjuvant chemotherapy (vs. none) & $2.25(1.70-2.99)$ & $<0.001$ & $1.11(0.73-1.70)$ & 0.632 \\
\hline Stage II (vs. stage I) & $2.23(1.56-3.19)^{\mathrm{a}}$ & $<0.001^{\mathrm{a}}$ & $1.86(1.17-2.95)^{\mathrm{a}}$ & $0.009^{\mathrm{a}}$ \\
\hline Stage III (vs. stage I) & $4.15(2.89-5.96)^{\mathrm{a}}$ & $<0.001^{\mathrm{a}}$ & $3.01(1.87-4.84)^{\mathrm{a}}$ & $<0.001^{\mathrm{a}}$ \\
\hline HER2 amplification (vs. negative) & $1.04(0.60-1.78)$ & 0.899 & & \\
\hline Ever neutropeniab (vs. never neutropenia) & $4.74(2.93-7.67)^{\mathrm{a}}$ & $<0.001^{\mathrm{a}}$ & $3.51(2.08-5.93)^{\mathrm{a}}$ & $<0.001^{\mathrm{a}}$ \\
\hline
\end{tabular}

ALC, absolute lymphocyte count; OR, odds ratio; CI, confidence interval; ECOG, Eastern Cooperative Oncology Group; HER2, epidermal growth factor receptor 2 .

${ }^{a}$ Statistically significant variables.

${ }^{\mathrm{b}}$ Definition of ever neutropenia: absolute neutrophil count $<1,000 / \mu \mathrm{L}$.

Table 3. Multivariable analysis of the clinical characteristics and ever lymphopenia for OM or GCM in patients with gastric cancer $(\mathbf{n}=\mathbf{1 , 2 2 2})$

\begin{tabular}{|c|c|c|c|c|}
\hline \multirow{2}{*}{ Variable } & \multicolumn{2}{|l|}{$\mathrm{OM}$} & \multicolumn{2}{|l|}{ GCM } \\
\hline & $\operatorname{HR}(95 \% \mathrm{CI})$ & $p$ value & $\operatorname{HR}(95 \% \mathrm{CI})$ & $p$ value \\
\hline Age at diagnosis, /yr & $1.05(1.02-1.07)^{\mathrm{a}}$ & $<0.001^{\mathrm{a}}$ & $1.03(1.00-1.05)^{\mathrm{a}}$ & $0.030^{\mathrm{a}}$ \\
\hline ECOG 2 (vs. ECOG o-1) & $1.61(0.81-3.22)$ & 0.177 & $1.18(0.51-2.72)$ & 0.700 \\
\hline Received adjuvant chemotherapy (vs. none) & $0.50(0.29-0.88)^{\mathrm{a}}$ & $0.017^{\mathrm{a}}$ & $0.37(0.20-0.66)^{\mathrm{a}}$ & $0.001^{\mathrm{a}}$ \\
\hline Stage II (vs. stage I) & $5.57(2.49-12.5)^{\mathrm{a}}$ & $<0.001^{\mathrm{a}}$ & $18.8(5.55-63.8)^{\mathrm{a}}$ & $<0.001^{\mathrm{a}}$ \\
\hline Stage III (vs. stage I) & $33.8(17.1-66.7)^{\mathrm{a}}$ & $<0.001^{\mathrm{a}}$ & $158.7(53 \cdot 5-471)^{\mathrm{a}}$ & $<0.001^{\mathrm{a}}$ \\
\hline Ever lymphopenia (vs. never lymphopenia) & $1.83(1.17-2.85)^{\mathrm{a}}$ & $0.008^{\mathrm{a}}$ & $1.58(0.96-2.60)^{\mathrm{a}}$ & $0.048^{a}$ \\
\hline
\end{tabular}

OM, overall mortality; GCM, gastric cancer-specific mortality; HR, hazard ratio; CI, confidence interval; ECOG, Eastern Cooperative Oncology Group.

${ }^{\mathrm{a}}$ Statistically significant variables.

er stage (stage III vs. I: HR, 70.3; 95\% CI, 33.7 to $146 ; p<$ 0.001) and ever lymphopenia (HR, 1.83; 95\% CI, 1.18 to 2.81; $p=0.006)$ were associated with shorter DFS after multivariate analysis (Table 4 and Fig. 2A). In contrast to the univariate analysis, patients who received adjuvant chemotherapy achieved longer DFS (HR, o.36; 95\% CI, 0.21 to $0.63 ; p<0.001$ ) (Table 4 ) compared to those who did not. Analysis of the association between ever lymphopenia and DFS in patients stratified by cancer stage revealed that ever lymphopenia was associated with recurrence not only in those with stage I gastric cancer (stage I: HR, 3.26; 95\% CI, 1.04 to 10.18; $p=0.042$ ) (Fig. 2B), but also in those with stage II-III (HR, 1.84; 95\% CI, 1.16 to $2.92 ; p=0.009$ ) (Fig. $2 \mathrm{C}$ ). The 5 -year cumulative recurrence rates were $15.1 \%$ and $4.6 \%$ for ever lymphopenic and never lymphopenic patients, respectively (Fig. 2A).

\section{DISCUSSION}

We found that the post-diagnosis peripheral ALC was associated with OM, GCM, and DFS in patients with stage I-III gastric cancer. Notably, the association between ever lymphopenia and mortality persisted over time. The increasing divergence of the survival curves over time was consistent with the results of previous 

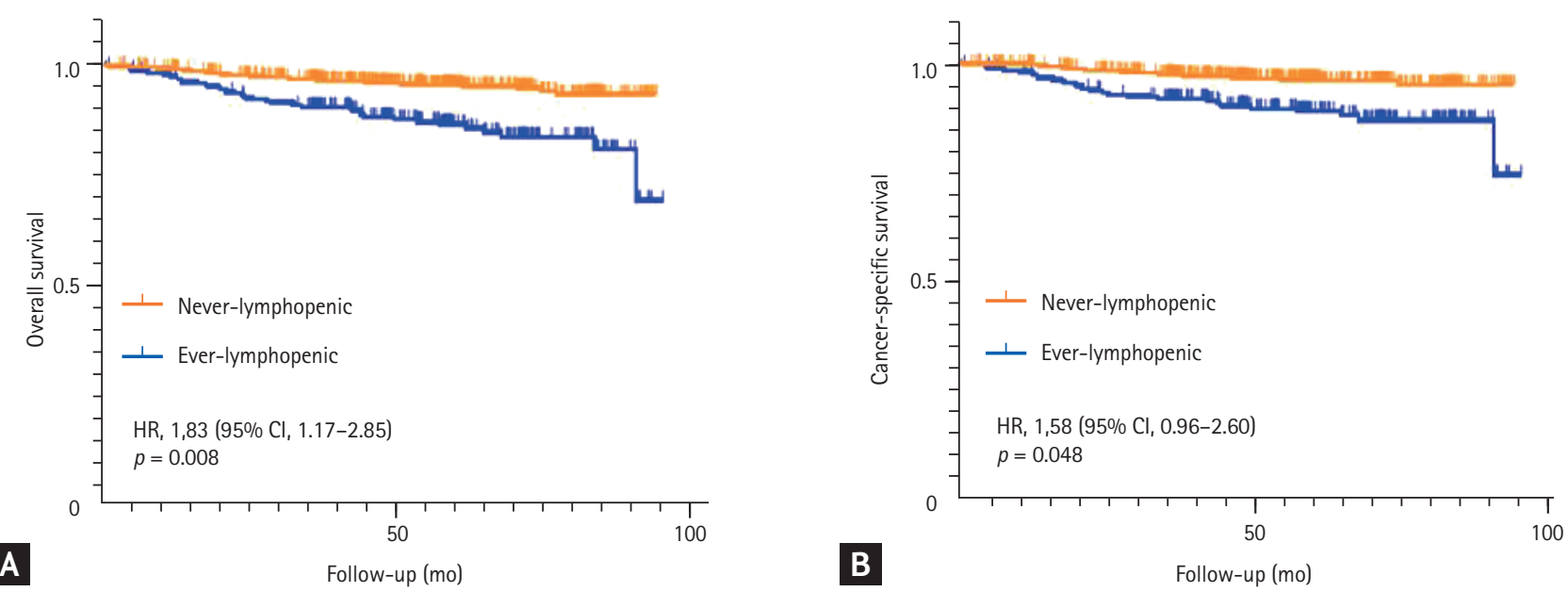

Figure 1. Kaplan-Meier curves showing (A) overall survival and (B) gastric cancer-specific survival according to the ever lymphopenia (lymphopenia defined as absolute lymphocyte count $<1,000 / \mu \mathrm{L}$ ). HR, hazard ratio; CI, confidence interval.

Table 4. Multivariable analysis of the clinical characteristics and ever lymphopenia for DFS in patients with gastric cancer $(\mathrm{n}=1,222)$

\begin{tabular}{|c|c|c|c|c|}
\hline \multirow{3}{*}{ Variable } & \multicolumn{4}{|c|}{ DFS } \\
\hline & \multicolumn{2}{|c|}{ Univariate analysis } & \multicolumn{2}{|c|}{ Multivariate analysis } \\
\hline & $\operatorname{HR}(95 \% \mathrm{CI})$ & $p$ value & $\operatorname{HR}(95 \% \mathrm{CI})$ & $p$ value \\
\hline Age at diagnosis, /yr & $1.03(1.02-1.05)$ & 0.001 & $1.02(1.00-1.04)$ & 0.105 \\
\hline ECOG 2 (vs. ECOG o-1) & $2.85(1.38-5.89)$ & 0.005 & $0.73(0.32-1.66)$ & 0.446 \\
\hline Received adjuvant chemotherapy (vs. none) & $3.69(2.42-5.63)^{\mathrm{a}}$ & $<0.001^{\mathrm{a}}$ & $0.36(0.21-0.63)^{\mathrm{a}}$ & $<0.001^{\mathrm{a}}$ \\
\hline Stage II (vs. stage I) & $6.21(2.87-13.4)^{\mathrm{a}}$ & $<0.001^{\mathrm{a}}$ & $10.4(4.54-23.8)^{\mathrm{a}}$ & $<0.001^{\mathrm{a}}$ \\
\hline Stage III (vs. stage I) & $40.4(21.7-75.2)^{\mathrm{a}}$ & $<0.001^{\mathrm{a}}$ & $70.3(33.7-146)^{\mathrm{a}}$ & $<0.001^{\mathrm{a}}$ \\
\hline Ever lymphopenia (vs. never lymphopenia) & $3.67(2.41-5.60)^{\mathrm{a}}$ & $<0.001^{\mathrm{a}}$ & $1.83(1.18-2.81)^{\mathrm{a}}$ & $0.006^{\mathrm{a}}$ \\
\hline
\end{tabular}

DFS, disease-free survival; HR, hazard ratio; CI, confidence interval; ECOG, Eastern Cooperative Oncology Group.

${ }^{\mathrm{a}}$ Statistically significant variables.

studies that showed the progressive separation of the survival curves of patients with surgically resected triple-negative breast cancer or pancreatic cancer grouped by their lymphopenic status $[15,20]$. These results suggest that lymphopenic status, as a surrogate for host immunity, plays an important role in the prognosis of patients with surgically resected gastric cancer.

The prognostic effect of the ALC in patients with gastric cancer has not been adequately investigated, as most previous studies focused on the prognostic value of the NLR instead [21]. Several studies have demonstrated the association between systemic inflammation and cancer progression, and the NLR reflects systemic inflammatory status [22]. However, the NLR has some limitations as a prognostic factor. The NLR is composed of two potentially independent biological factors, neutrophils and lymphocytes. Neutrophils are associated with inflammation, whereas lymphocytes are involved in host immunity, and it is difficult to identify which is more strongly associated with prognosis. In addition to the NLR, other biological markers related to systemic inflammation, such as C-reactive protein level [23], the platelet-to-lymphocyte ratio [24], and albumin level [25], have been associated with prognosis in gastric cancer. The ALC is a surrogate marker of host immunity and we chose to investigate the relationship between prognosis and host immunity, represented by the ALC, rather than a marker of systemic inflammation, such as the NLR.

Previous studies have shown that the ALC or absolute monocyte count at the time of diagnosis correlates with 


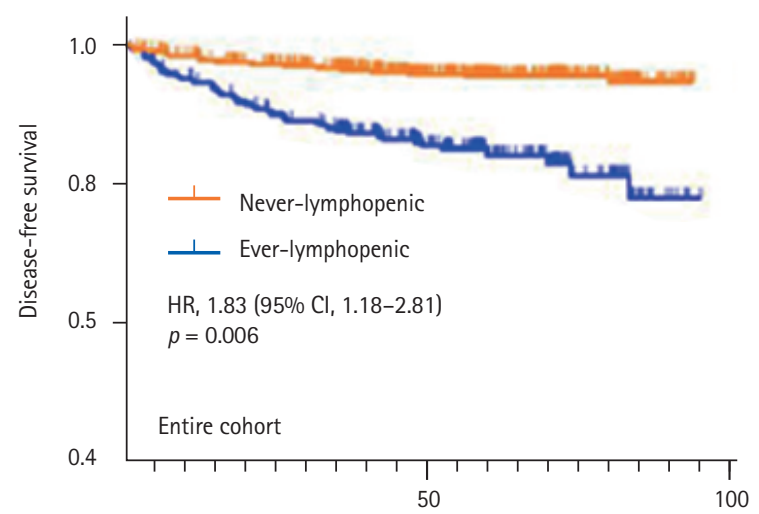

A Follow-up (mo)

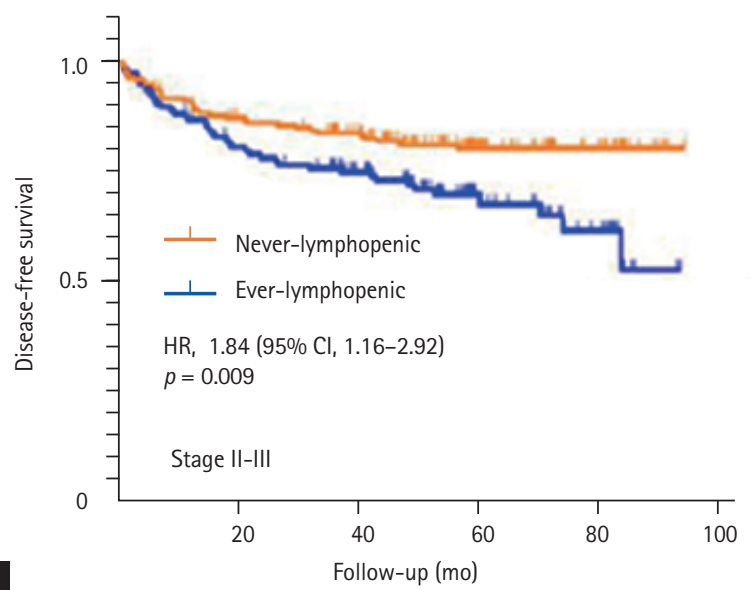

C

prognosis $[11,18,26]$; however, to our knowledge, ours is the first study to show a significant association between ever lymphopenia and cancer-specific mortality in patients with potentially curable gastric cancer. Additionally, ever lymphopenia was significantly associated with recurrence. Our data suggest that the presence of lymphopenia not only at the time of diagnosis, but also before, during, and after therapy is associated with the prognosis of patients with gastric cancer.

Several factors, including higher cancer stage, older age at diagnosis, and ever neutropenia, were positively associated with ever lymphopenia. Higher cancer stage was associated with baseline lymphopenia as well as ever lymphopenia. These results are consistent with those of previous studies that found that larger tumor burdens were correlated with lower lymphocyte counts [27]. Notably, tumor cells induce the synthesis of immunosuppressive molecules, which may lead to lymphopenia and immune evasion [28]. Taken together, the evidence

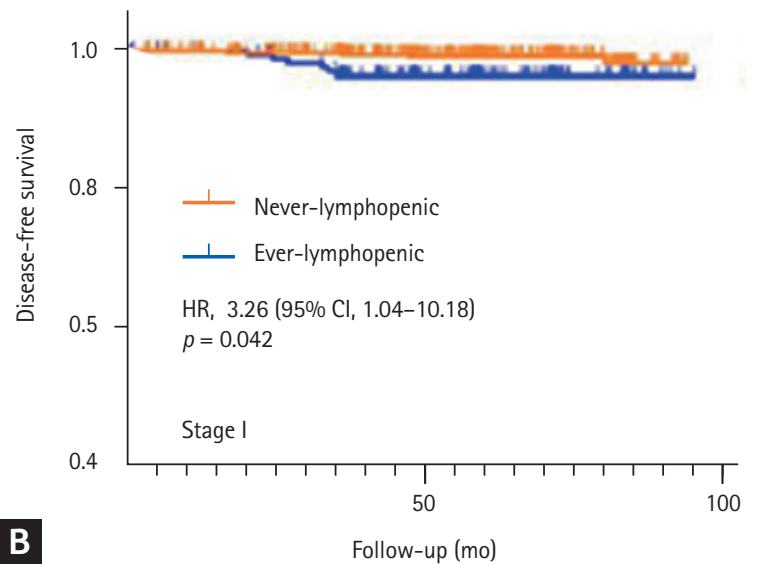

Figure 2. Kaplan-Meier curves of disease-free survival for the two groups according to their lymphopenic status. (A) Disease-free survival in the entire cohort according to lymphopenic status. (B, C) Disease-free survival of patients with stage I and II-III gastric cancer according to their lymphopenic status. HR, hazard ratio; CI, confidence interval.

suggests that the occurrence of tumor-induced immune suppression promotes lymphopenia, which in turn complicates cancer therapy.

According to our multivariate analyses that incorporated known risk factors for poor prognosis, ever lymphopenia was independently correlated with higher cancer-specific mortality and shorter DFS in patients with surgically resected gastric cancer. Although the mechanism for the association between ever lymphopenia and prognosis is not clear, possible explanations include (1) ever lymphopenia may be associated with a pre-existing immunosuppression-associated condition in the host, resulting in an inadequate immunologic response to malignancy; and (2) ever lymphopenia may be a consequence of lympholytic cytokines produced by the tumor cells [29,30]. Lymphopenia caused by tumor cells may weaken the host's immune response to cancer; moreover, the tumor cells can alter the homeostasis of lymphocyte pools in patients with cancer [31]. 
A recent study showed that the specific nature of infiltrating immune cells could affect the prognoses of patients with various cancers [32]. A number of potentially promising immune biomarkers have been discovered, including TILs [33], circulating tumor-specific T cells [34], differentiated CD8+ T cells [35], and regulatory $\mathrm{T}$ cell accumulation [36]; however, most of these are yet to be validated. Moreover, a meta-analysis found that high expression of TILs, particularly CD8+ T cells, could be a potential indicator of improved prognosis [37]. High expression of TILs was also reported to significantly improve the prognoses of patients with gastric cancer who had high microsatellite instability or were positive for Epstein-Barr virus infection, as these features increased the tumors' sensitivity to immune checkpoint inhibitors [38].

In a previous study, an increased number of TILs in patients with triple-negative breast cancer was found to be directly correlated with increased peripheral lymphocyte counts during neoadjuvant chemotherapy [15]. Additionally, elevated peripheral lymphocyte counts in patients with rectal cancer were significantly correlated with high tissue levels of $\mathrm{CD}_{4}+$ and, CD8+ T cells [16]. As such, peripheral lymphocyte counts may also be associated with TILs in patients with gastric cancer, and may also predict immunological responses to cancer. Immune response measures, such as peripheral lymphocyte counts, could serve as predictors of prognosis or as surrogate endpoints of clinical responses to immunotherapy.

There were several limitations to our study. First, it was a retrospective analysis conducted at a single institution; therefore, multi-center, randomized studies are required to verify the prognostic significance of ever lymphopenia. Second, we used a threshold level of 1,000 lymphocytes per microliter as a cut-off value for prognosis; however, various cut-off values have previously been used when evaluating the prognostic significance of ALCs, and additional studies are required to establish the optimal threshold. Third, the HR of higher cancer stage was overestimated compared to those of previous studies because our cohort had a relatively larger number of stage IA patients (stage IA vs. IB: 772 vs. 133). Fourth, we cannot rule out the possibility that patients with poorer prognoses had their blood counts measured more often. Finally, we did not evaluate any differences in prognosis regarding the timing of lymphopenia after surgery.

This study can serve as a pilot study for future prospective studies investigating the association between ever lymphopenia within a specific timeframe and prognosis for gastric cancer. According to a previous study, $60 \%$ to $70 \%$ of gastric cancer recurs within 2 years after surgery [39]; therefore, the prognostic effect of lymphopenia within 2 years after surgery should be evaluated in these patients. Understanding the mechanisms involved in lymphopenia, as well as the consequences thereof on patient outcomes, may facilitate the development of strategies to improve patient survival. Further studies should be conducted to evaluate whether peripheral lymphocytes are correlated with TILs in gastric cancer, as well as to identify which changes in specific subsets of peripheral lymphocytes affect the prognosis of gastric cancer.

In conclusion, we found a persistent association between peripheral lymphocyte counts and cancer-specific mortality and $\mathrm{OM}$ in patients with potentially curable gastric cancer. These results suggest that immune functions may play a key role in the prognosis of this disease. In the future, we plan to evaluate the roles of peripheral lymphocyte counts and TILs as potential prognostic factors in patients with gastric cancer.

\section{KEY MESSAGE}

1. On multivariate analysis, older age at diagnosis, higher cancer stage, and ever neutropenia were significantly associated with ever lymphopenia.

2. The post-diagnosis peripheral absolute lymphocyte counts were found to be independent prognostic indicators for gastric cancer-specific mortality and disease-free survival in patients with stage I-III gastric cancer who had curative gastrectomy.

3. Notably, the divergence of the survival curves depending on lymphopenic status appeared to strengthen over time.

\section{Conflict of interest}

No potential conflict of interest relevant to this article was reported. 


\section{Acknowledgments}

Statistical consultation was supported by the Department of Biostatics of the Catholic University Research Coordinating Center.

\section{REFERENCES}

1. Crew KD, Neugut AI. Epidemiology of upper gastrointestinal malignancies. Semin Oncol 2004;31:450-464.

2. Washington $\mathrm{K}$. 7th Edition of the AJCC cancer staging manual: stomach. Ann Surg Oncol 2010;17:3077-3079.

3. Siewert JR, Bottcher K, Stein HJ, Roder JD. Relevant prognostic factors in gastric cancer: ten-year results of the German Gastric Cancer Study. Ann Surg 1998;228:449-461.

4. Campian JL, Sarai G, Ye X, Marur S, Grossman SA. Association between severe treatment-related lymphopenia and progression-free survival in patients with newly diagnosed squamous cell head and neck cancer. Head Neck 2014;36:1747-1753.

5. Manuel M, Tredan O, Bachelot T, et al. Lymphopenia combined with low TCR diversity (divpenia) predicts poor overall survival in metastatic breast cancer patients. Oncoimmunology 2012;1:432-440.

6. Vicente Conesa MA, Garcia-Martinez E, Gonzalez Billalabeitia E, et al. Predictive value of peripheral blood lymphocyte count in breast cancer patients treated with primary chemotherapy. Breast 2012;21:468-474.

7. Ray-Coquard I, Cropet C, Van Glabbeke M, et al. Lymphopenia as a prognostic factor for overall survival in advanced carcinomas, sarcomas, and lymphomas. Cancer Res 2009;69:5383-5391.

8. Balmanoukian A, Ye X, Herman J, Laheru D, Grossman SA. The association between treatment-related lymphopenia and survival in newly diagnosed patients with resected adenocarcinoma of the pancreas. Cancer Invest 2012;30:571-576.

9. Lissoni P, Brivio F, Fumagalli L, et al. Efficacy of cancer chemotherapy in relation to the pretreatment number of lymphocytes in patients with metastatic solid tumors. Int J Biol Markers 2004;19:135-140.

10. Bruckner HW, Lavin PT, Plaxe SC, Storch JA, Livstone EM. Absolute granulocyte, lymphocyte, and moncyte counts. Useful determinants of prognosis for patients with metastatic cancer of the stomach. JAMA 1982;247:1004-1006.
11. Eo WK, Jeong DW, Chang HJ, et al. Absolute monocyte and lymphocyte count prognostic score for patients with gastric cancer. World J Gastroenterol 2015;21:2668-2676.

12. Hu ZD, Huang YL, Qin BD, et al. Prognostic value of neutrophil to lymphocyte ratio for gastric cancer. Ann Transl Med 2015;3:50.

13. Miyamoto R, Inagawa S, Sano N, Tadano S, Adachi S, Yamamoto $M$. The neutrophil-to-lymphocyte ratio (NLR) predicts short-term and long-term outcomes in gastric cancer patients. Eur J Surg Oncol 2018;44:607-612.

14. Mori M, Shuto K, Kosugi C, et al. An increase in the neutrophil-to-lymphocyte ratio during adjuvant chemotherapy indicates a poor prognosis in patients with stage II or III gastric cancer. BMC Cancer 2018;18:1261.

15. Afghahi A, Purington N, Han SS, et al. Higher absolute lymphocyte counts predict lower mortality from early-stage triple-negative breast cancer. Clin Cancer Res 2018;24:2851-2858.

16. Shorbagy SE, Elfarargy OM, Salem RA, et al. Peripheral and tissue lymphocytes as predictors of pathological response in locally advanced rectal cancer post neoadjuvant chemoradiotherapy. J Cancer Ther 2017;8:250-267.

17. Lee KH, Kim EY, Yun JS, et al. The prognostic and predictive value of tumor-infiltrating lymphocytes and hematologic parameters in patients with breast cancer. BMC Cancer 2018;18:938.

18. Tatara T, Suzuki S, Kanaji S, et al. Lymphopenia predicts poor prognosis in older gastric cancer patients after curative gastrectomy. Geriatr Gerontol Int 2019;19:1215-1219.

19. Japanese Gastric Cancer Association. Japanese gastric cancer treatment guidelines 2014 (ver. 4). Gastric Cancer 2017;20:1-19.

20. d'Engremont C, Vernerey D, Pointet AL, et al. Additive value of pre-operative and one-month post-operative lymphocyte count for death-risk stratification in patients with resectable pancreatic cancer: a multicentric study. BMC Cancer 2016;16:823.

21. Szor DJ, Dias AR, Pereira MA, et al. Prognostic role of neutrophil/lymphocyte ratio in resected gastric cancer: a systematic review and meta-analysis. Clinics (Sao Paulo) 2018;73:e360.

22. Coussens LM, Werb Z. Inflammation and cancer. Nature 2002;420:860-867.

23. Yu Q, Yu XF, Zhang SD, Wang HH, Wang HY, Teng LS. Prognostic role of C-reactive protein in gastric cancer: a meta-analysis. Asian Pac J Cancer Prev 2013;14:5735-5740. 
24. Lee S, Oh SY, Kim SH, et al. Prognostic significance of neutrophil lymphocyte ratio and platelet lymphocyte ratio in advanced gastric cancer patients treated with FOLFOX chemotherapy. BMC Cancer 2013;13:350.

25. Powell AGMT, Parkinson D, Patel N, Chan D, Christian A, Lewis WG. Prognostic significance of serum inflammatory markers in gastric cancer. J Gastrointest Surg 2018;22:595-605.

26. Feng F, Zheng G, Wang Q, et al. Low lymphocyte count and high monocyte count predicts poor prognosis of gastric cancer. BMC Gastroenterol 2018;18:148.

27. Tang C, Liao Z, Gomez D, et al. Lymphopenia association with gross tumor volume and lung $\mathrm{V}_{5}$ and its effects on non-small cell lung cancer patient outcomes. Int J Radiat Oncol Biol Phys 2014;89:1084-1091.

28. Huang B, Zhao J, Unkeless JC, Feng ZH, Xiong H. TLR signaling by tumor and immune cells: a double-edged sword. Oncogene 2008;27:218-224.

29. Saito T, Kuss I, Dworacki G, Gooding W, Johnson JT, Whiteside TL. Spontaneous ex vivo apoptosis of peripheral blood mononuclear cells in patients with head and neck cancer. Clin Cancer Res 1999;5:1263-1273.

30. Dworacki G, Meidenbauer N, Kuss I, et al. Decreased zeta chain expression and apoptosis in $\mathrm{CD}_{3}+$ peripheral blood $\mathrm{T}$ lymphocytes of patients with melanoma. Clin Cancer Res 2001;7:947s-957s.

31. Goldrath AW, Bevan MJ. Selecting and maintaining a diverse T-cell repertoire. Nature 1999;402:255-262.
32. Fridman WH, Pages F, Sautes-Fridman C, Galon J. The immune contexture in human tumours: impact on clinical outcome. Nat Rev Cancer 2012;12:298-306.

33. Naito Y, Saito K, Shiiba K, et al. CD8+ T cells infiltrated within cancer cell nests as a prognostic factor in human colorectal cancer. Cancer Res 1998;58:3491-3494.

34. Britten CM, Janetzki S, van der Burg SH, et al. Minimal information about $\mathrm{T}$ cell assays: the process of reaching the community of $\mathrm{T}$ cell immunologists in cancer and beyond. Cancer Immunol Immunother 2011;60:15-22.

35. Czystowska M, Gooding W, Szczepanski MJ, et al. The immune signature of $\mathrm{CD} 8(+) \mathrm{CCR}_{7}(+) \mathrm{T}$ cells in the peripheral circulation associates with disease recurrence in patients with HNSCC. Clin Cancer Res 2013;19:889-899.

36. Whiteside TL. What are regulatory T cells (Treg) regulating in cancer and why? Semin Cancer Biol 2012;22:327334 .

37. Lee JS, Won HS, Sun S, Hong JH, Ko YH. Prognostic role of tumor-infiltrating lymphocytes in gastric cancer: a systematic review and meta-analysis. Medicine (Baltimore) 2018;97:e11769.

38. Kang BW, Kim JG, Lee IH, Bae HI, Seo AN. Clinical significance of tumor-infiltrating lymphocytes for gastric cancer in the era of immunology. World J Gastrointest Oncol 2017;9:293-299.

39. Kang WM, Meng QB, Yu JC, Ma ZQ, Li ZT. Factors associated with early recurrence after curative surgery for gastric cancer. World J Gastroenterol 2015;21:5934-5940. 
Park SJ, et al. Absolute lymphocytes and gastric cancer

Supplementary Table 1. Univariate and multivariate analyses of predictors of baseline lymphopenia $(\mathrm{ALC}<1,000 / \mu \mathrm{L}, \mathrm{n}=1,222)$

\begin{tabular}{|c|c|c|c|c|}
\hline \multirow{3}{*}{ Variable } & \multicolumn{4}{|c|}{ Baseline lymphopenia } \\
\hline & \multicolumn{2}{|c|}{ Univariate analysis } & \multicolumn{2}{|c|}{ Multivariate analysis } \\
\hline & OR (95\% CI) & $p$ value & OR $(95 \% \mathrm{CI})$ & $p$ value \\
\hline Age at diagnosis, /yr & $1.02(1.00-1.04)$ & 0.118 & & \\
\hline ECOG 2 (vs. ECOG o-1) & $2.99(1.22-7.34)$ & 0.017 & $1.96(0.70-5.44)$ & 0.199 \\
\hline Stage II (vs. stage I) & $1.45(0.65-3.22)$ & 0.362 & $1.43(0.64-3.17)$ & 0.386 \\
\hline Stage III (vs. stage I) & $4.04(2.19-7.45)^{\mathrm{a}}$ & $<0.001^{\mathrm{a}}$ & $3.81(2.05-7.09)^{\mathrm{a}}$ & $<0.001^{\mathrm{a}}$ \\
\hline HER2 amplification (vs. negative) & $0.85(0.26-2.81)$ & 0.793 & & \\
\hline
\end{tabular}

ALC, absolute lymphocyte count; OR, odds ratio; CI, confidence interval; ECOG, Eastern Cooperative Oncology Group; HER2, epidermal growth factor receptor 2 .

${ }^{\text {a }}$ Statistically significant variables. 
Supplementary Table 2. Incidence of ever lymphopenia (ALC $<1,000 / \mu \mathrm{L}, \mathrm{n}=1,222)$ according to cancer stage and neutropenia

\begin{tabular}{lc}
\hline Variable & Ever lymphopenia (\%) \\
\hline Stage I & $172 / 905(19.0)$ \\
Stage II & $58 / 169(34.3)$ \\
Stage III & $73 / 148(49.3)$ \\
Ever neutropenia $^{a}$ & $43 / 74(58.1)$ \\
Never neutropenia $^{2}$ & $260 / 1,148(22.6)$ \\
\hline
\end{tabular}

ALC, absolute lymphocyte count.

${ }^{a}$ Definition of ever neutropenia: absolute neutrophil count < $1,000 / \mu \mathrm{L}$. 